\title{
Fatores de Risco para Acidente Vascular Encefálico após Cirurgia de Revascularização do Miocárdio
}

\author{
Risk Factors for Stroke after Coronary Artery Bypass Grafting \\ Dinaldo Cavalcanti de Oliveira, Carlos Romerio Ferro, João Bosco de Oliveira, Marcelo Menezes Malta, Plínio Barros \\ Neto, Silvia J. F. Cano, Stevan Krieker Martins, Luis Carlos B. Souza, Adib Domingos Jatene, Leopoldo Soares Piegas \\ Hospital do Coração - Associação do Sanatório Sírio, São Paulo, SP - Brasil
}

\section{Resumo}

Fundamento: O acidente vascular encefálico (AVE) é uma temida complicação após cirurgia de revascularização do miocárdio (CRM), com incidência entre 1,3\% e 4,3\%.

Objetivo: Identificar fatores preditores de AVE após CRM, na era moderna da cirurgia cardíaca.

Métodos: Este é um estudo caso-controle de 65 pares de pacientes, no qual o pareamento foi realizado por sexo, idade ( \pm 3 anos) e data da CRM ( \pm 3 meses). Os casos são pacientes submetidos à CRM eletiva com circulação extracorpórea (CEC), que apresentaram AVE (definido como déficit clínico neurológico até 24 horas de pós-operatório e confirmado por exame de imagem), e os controles aqueles submetidos à CRM eletiva com CEC sem AVE.

Resultados: A análise univariada revelou que o número de vasos revascularizados foi associado com a ocorrência de AVE após a CRM ( $3 \pm 0,8$ vs. 2,76 $\pm 0,8, p=0,01)$. Na análise multivariada por regressão logística condicional, a hipertensão arterial sistêmica [OR: 6,1 (1,5-24), $p=0,009]$ e o diabete melito [OR: $3,1(1,09-11), p=0,03]$ foram determinantes de maior chance de AVE após CRM, e o infarto agudo do miocárdio > 1 mês determinante de menor chance [OR: $0,1(0,03-0,36), p=0,003]$.

Conclusão: Hipertensão e diabete melito foram identificados como preditores independentes de AVE nas primeiras 24 horas de pós-operatório de CRM. Em pacientes com tais fatores de risco, é possível que o conhecimento dos mecanismos causadores da injúria cerebral represente uma estratégia capaz de diminuir a incidência de AVE após CRM. (Arq Bras Cardiol 2008;91(4):234-237)

Palavras-chave: Acidente cerebrovascular, revascularização miocárdica / efeitos adversos, fatores de risco.

\author{
Summary \\ Background: Stroke is a feared complication after coronary artery bypass grafting surgery (CABG), with an incidence between 1.3 and $4.3 \%$.
}

Objective: To identify predictive factors for stroke after CABG in the modern era of cardiac surgery.

Methods: This is a case-control study of 65 pairs of patients, paired by sex, age ( \pm 3 years) and date of CABG ( \pm 3 months). The cases were patients submitted to elective CABG with extracorporeal circulation (ECC) that presented stroke (defined as clinical neurological deficit up to 24 hours post-operatively and confirmed by imaging assessment) and the controls were those individuals submitted to elective CABG with ECC, but without stroke.

Results: The univariate analysis demonstrated that the number of revascularized vessels was associated with the occurrence of stroke after the CABG ( $3 \pm 0.8$ vs. $2.76 \pm 0.8, p=0.01)$. The multivariate analysis by conditional logistic regression showed that systemic arterial hypertension (SAH) [OR: $6.1(1.5-24), p=0.009]$ and diabete mellitus (DM) [OR: $3.1(1.09-11), p=0.03]$ were the determinants of the highest chance of stroke after $C A B G$, whereas acute myocardial infarction (AMI) > 1 month, was the determinant of the lowest chance of stroke [OR: 0.1 (0.03 $-0.36), p=0.003]$.

Conclusion: Hypertension and diabete mellitus were identified as independent predictors of stroke within the first 24 postoperative hours after CABG. In patients with such risk factors, it is possible that the knowledge of the causal mechanisms of brain injury represents a strategy capable of decreasing the incidence of stroke after CABG. (Arq Bras Cardiol 2008;91(4):213-216)

Key words: Cerebrovascular accident; myocardial revascularization / adverse effects; risk factors.

Full texts in English - http://www.arquivosonline.com.br

Correspondência: Dinaldo Cavalcanti de Oliveira •

Rua Abílio Soares, 625 / 64 A - Paraíso - 04005-002 - São Paulo, SP - Brasil

E-mail: dinaldo@cardiol.br

Artigo enviado em 29/11/2007; revisado recebido em 15/02/08; aceito em 15/02/08. 


\section{Introdução}

A cirurgia de revascularização do miocárdio (CRM) é um tratamento eficaz em prolongar e melhorar a vida de pacientes com doença arterial coronariana (DAC) ${ }^{1}$.

O acidente vascular encefálico (AVE) é uma temida complicação dessa cirurgia. Sua incidência, na década de 1960, era de até 9\%²; e a despeito da melhora das técnicas cirúrgicas dos últimos anos, essa complicação tem atualmente incidência que varia de $1,3 \%$ a $4,3 \%{ }^{3-6}$.

Estudos clínicos prévios identificaram preditores de AVE após CRM, porém a baixa freqüência desse evento, a nãouniformidade na avaliação de variáveis relacionadas à injúria cerebral e o tamanho das amostras são fatores limitantes desses estudos ${ }^{7-9}$.

Acreditamos que análises de fatores de risco contemporâneos são necessárias para determinar quais são realmente os preditores de AVE na era moderna da cirurgia cardíaca.

Realizamos um estudo caso-controle a fim de identificar fatores preditores de AVE após CRM.

\section{Métodos}

Este é um estudo caso-controle de 65 pares de pacientes, no qual o pareamento foi realizado por sexo, idade $( \pm 3$ anos) e data da CRM ( \pm 3 meses). Os casos são pacientes (pts) submetidos à primeira CRM eletiva com circulação extracorpórea (CEC), que apresentaram AVE (definido como déficit clínico neurológico até 24 horas de pós-operatório e confirmado por exame de imagem), e os controles aqueles também submetidos à primeira CRM eletiva com CEC que não sofreram AVE.

As características clínicas e cirúrgicas avaliadas foram: hipertensão arterial sistêmica (HAS), diabete melito (DM), dislipidemia (DLP), tabagismo, AVE prévio, lesão de carótida prévia ( $\geq 60 \%$ ), fibrilação atrial pregressa, função ventricular (definida pela fração de ejeção do ventrículo esquerdo (FEVE) ao ecocardiograma em: normal se FEVE $>60 \%$, discreta entre $50 \%$ e $60 \%$, moderada de $40 \%$ a $50 \%$ e grave $<40 \%$ ), número de vasos revascularizados, tempo de CEC, insuficiência renal crônica (creatinina $\geq 1,5 \mathrm{mg} / \mathrm{dl}$ ), infarto agudo do miocárdio (IAM) $\leq 1$ mês e > 1 mês, intervenção coronária percutânea (ICP) prévia.

As variáveis contínuas foram descritas em média e desvio padrão, enquanto as categóricas em valores percentuais.

Na análise univariada, utilizamos o teste da homogeneidade marginal para avaliação das variáveis categóricas e o teste t de Student para as variáveis numéricas. Para o cálculo da razão de chance $(\mathrm{RC})$, realizamos análise multivariada por regressão logística condiconal, sendo $p$ considerado significativo quando $\leq 0,05$.

As variáveis avaliadas no modelo multivariado de regressão logística condicional (utilizada para avaliar a probabilidade de as variáveis determinarem os casos) foram aquelas que nos modelos univariados apresentaram $p$-valores $\leq 0,1$ ou que tenham sido consideradas relevantes clinicamente.

Este estudo foi aprovado pelo comitê de ética em pesquisa da nossa instituição.

\section{Resultados}

No período de janeiro de 2000 a abril de 2006, foram submetidos à primeira CRM eletiva com CEC 3.420 pacientes, $\mathrm{e}$ 65 (1,9\%) apresentaram AVE, conforme definição do estudo.

Incluímos 65 pares de pacientes, de acordo com o delineamento do estudo, sendo 41 de homens e 24 de mulheres. A idade média foi 70,6 2 8,1 anos.

A freqüência de distribuição das variáveis analisadas e suas associações com a ocorrência de AVE, de acordo com análise univariada, são demonstradas nas tabelas 1 e 2. As variáveis que tiveram associação significativa com AVE, no pós-operatório de revascularização do miocárdio, foram o maior número de vasos revascularizados e a ICP prévia.

O modelo final da análise multivariada por regressão logística condicional incluiu as seguintes variáveis: HAS, DM, DLP, tabagismo, AVE prévio, lesão de carótida prévia, fibrilação atrial pregressa, função ventricular, número de vasos revascularizados, tempo de CEC, IAM $\leq 1$ mês e $>1$ mês e ICP prévia.

Após controle para esses fatores, identificamos a HAS [RC: $6,1(1,5-24), p=0,009]$ e o DM [RC: 3,1 (1,09-11), $p=$ $0,03]$ como fatores determinantes de maior chance de AVE no pós-operatório de RM, enquanto o IAM >1 mês [RC: 0,1 $(0,03-0,36), p=0,003]$ foi o fator associado à menor chance desse evento.

Tabela 1 - Características clínicas e de exames complementares dos pacientes

\begin{tabular}{lccc}
\hline Variáveis & $\begin{array}{c}\text { Caso } \\
(\mathrm{n}=65 \mathrm{pts})\end{array}$ & $\begin{array}{c}\text { Controle } \\
(\mathrm{n}=65 \mathrm{pts})\end{array}$ & p valor \\
\hline HAS $(\%)$ & 68 & 75 & 0,2 \\
\hline Diabete melito (\%) & 49 & 35 & 0,1 \\
\hline Dislipidemia (\%) & 52 & 58 & 0,4 \\
\hline Tabagismo (\%) & 28 & 24 & 0,6 \\
\hline AVE (\%) & 3 & 0 & 0,2 \\
\hline IRC (\%) & 8 & 6 & 0,7 \\
\hline EC $\geq 60 \%(\%)$ & 15 & 15 & 0,9 \\
\hline
\end{tabular}

HAS - hipertensão arterial sistêmica; AVE - acidente vascular encefálico; IRC insuficiência renal crônica; EC - estenose de artéria carótida; pts - pacientes.

\section{Tabela 2 - Perfil clínico dos pacientes}

\begin{tabular}{|c|c|c|c|}
\hline Variáveis & $\begin{array}{c}\text { Caso } \\
\text { (n=65 pts) }\end{array}$ & $\begin{array}{l}\text { Controle (n } \\
=65 \mathrm{pts} \text { ) }\end{array}$ & $p$ valor \\
\hline IAM $\leq 1$ mês (\%) & 15 & 18 & 0,6 \\
\hline IAM > 1 mês (\%) & 18 & 14 & 0,4 \\
\hline Fibrilação atrial (\%) & 3 & 1,5 & 0,5 \\
\hline $\operatorname{ICP}(\%)$ & 1,5 & 15 & 0,007 \\
\hline CRM (\%) & 12 & 12 & 0,9 \\
\hline FEVE de $40 \%$ a $50 \%$ (\%) & 17 & 23 & 0,4 \\
\hline FEVE $<40 \%(\%)$ & 7 & 9 & 0,3 \\
\hline
\end{tabular}




\section{Discussão}

Neste estudo, realizado na era contemporânea da CRM, identificamos dois fatores do perfil clínico dos pacientes, HAS e DM, que foram determinantes de maior chance de AVE no pós-operatório.

Os dois mecanismos responsáveis pelo AVE são a isquemia e a hemorragia ${ }^{10}$.

A isquemia pode ser causada por fenômenos embólicos (cardíaco, da artéria aorta ou de artérias proximais), tromboembolismo de vasos intra ou extracranianos e hipoperfusão sistêmica. A hemorragia ocorre associada à hipertensão arterial sistêmica ou à reperfusão de áreas infartadas ${ }^{10}$.

No AVE hemorrágico, o sangue acumula-se fora do vaso sangüíneo e pode contribuir para a elevação da pressão cerebral e a morte da célula neurológica ${ }^{10}$.

Na injúria isquêmica causada por embolia cardíaca, geralmente os êmbolos são originários do átrio, como na fibrilação atrial, ou do ventrículo esquerdo, como no IAM recente. Habitualmente, são múltiplos infartos predominantemente no território na artéria cerebral média. Quando ocorre tromboembolismo lacunar, a causa é aterosclerose induzida por hipertensão ou estenoses de artérias penetrantes no cérebro. O tromboembolismo trombótico é relacionado à aterosclerose de vasos intracranianos ou a anormalidades hematológicas, como aumento das hemácias ou plaquetas ${ }^{10}$.

O dano cerebral decorrente de hipoperfusão é causado por combinação de estenose extracraniana e hipotensão sistêmica, sendo mais freqüentemente unilateral, quando relacionado a embolismo, e bilateral, quando relacionado a importante e persistente hipotensão sistêmica ${ }^{10}$.

Quando o insulto cerebral tem dois ou mais mecanismos causadores, é classificado como de causas múltiplas, e aqueles sem mecanismos conhecidos são classificados como "outros"10.

Eventos neurológicos que ocorrem após CRM são classificados em tipo 1, quando são AVE focal, ataque isquêmico transitório ou dano cerebral fatal, e tipo 2, quando refletem injúria cerebral difusa com desorientação ou declínio intelectual normalmente reversível ${ }^{8}$.

O AVE, que ocorre nas primeiras 24 horas após a CRM, é uma complicação potencialmente catastrófica. Ele está associado ao aumento da mortalidade hospitalar, conforme demonstrado por Glance e cols. ${ }^{11}$ em estudo que avaliou 51.750 pacientes submetidos à revascularização cirúrgica do miocárdio, e, após ajuste para fatores de risco pré-operatório, o AVE determinou uma chance quatro vezes maior de óbitos ${ }^{12}$.

A identificação de fatores preditores de AVE e, quando possível, a atuação sobre eles, a fim de diminuir a incidência desse evento, são objetivos que pretendidos há mais de quatro décadas ${ }^{2}$. Nesse sentido, uma análise de 16.528 submetidos à CRM, nos anos 1990, revelou os seguintes preditores independentes: insuficiência renal crônica, IAM $<24$ horas, AVE prévio, doença carotídea, HAS, DM, idade > 75 anos,
FE $\leq 34 \%$, diminuição do débito cardíaco e fibrilação atrial no pós-operatório ${ }^{11}$.

Outros estudos também identificaram a HAS e o DM como preditores independentes de AVE no pós-operatório de CRM. Embora a rápida progressão de aterosclerose aórtica, carotídea, alterações trombóticas etc. estejam associadas à injúria cerebral, o verdadeiro mecanismo do AVE, em pacientes hipertensos e diabéticos, não está totalmente esclarecido e necessita de investigações futuras ${ }^{13-17}$.

As técnicas cirúrgicas e os cuidados pré, intra e pósoperatório têm sofrido mudanças nos últimos anos, contibuindo para a melhoria dos resultados da revascularização do miocárdio ${ }^{18}$. Entretanto, em razão dessas mudanças, não é possível inferir que todos os resultados de estudos clínicos realizados em outro momento da cirurgia cardíaca possam ser transpostos aos dias atuais.

A identificação de pacientes com características de maior risco para ocorrência de dano cerebral poderá permitir a adoção de medidas profiláticas fundamentadas no conhecimento prévio (conforme descrito anteriormente) dos mecanismos causadores de AVE.

Dessa forma, acreditamos que, em pacientes hipertensos e diabéticos, medidas como controle adequado da pressão arterial (evitando períodos de hipertensão e hipotensão), rastreamento de possíveis fontes emboligênicas cardíacas ou de outros territórios vasculares e a avaliação e manipulação criteriosa da artéria aorta possam contribuir para redução da incidência do AVE após a CRM.

\section{Conclusões}

A HAS e o DM foram determinantes de maior chance de AVE nas primeiras 24 horas de pós-operatório de CRM. Nos pacientes com tais fatores de risco, é possível que a atuação incisiva em mecanismos causadores de AVE contribua para redução de sua incidência.

\section{Agradecimentos}

Agradecemos aos profissionais de saúde da nossa instituição e às secretárias Val e Mônica, pois, sem a contribuição dessas pessoas, não seria possível realizar este estudo.

\section{Potencial Conflito de Interesses}

Declaro não haver conflito de interesses pertinentes.

\section{Fontes de Financiamento}

O presente estudo não teve fontes de financiamento externas.

\section{Vinculação Acadêmica}

Não há vinculação deste estudo a programas de pósgraduação. 


\section{Referências}

1. Palmer G, Herbert AM, Prince SL, Williams JL, Magee MJ, Brow P, et al. Coronary artery revascularization (CARE) registry: an observational study of on-pump and off-pump coronary artery revascularization. Ann Thorac Surg. 2007; 83: 986-92.

2. Mahanna EP, Blumenthal JA, White WD, Croughwell ND, Clancy CP, Smith $L R$, et al. Defining neuropsychological dysfunction after coronary bypass grafting. Ann Thorac Surg. 1996; 61: 1342-7.

3. Garden TJ, Hommefer PJ, Monolio TA, Pearson TA, Gott VL, Baumgartner WA, et al. Stroke following coronary artery bypass grafting: a ten year study. Ann Thorac Surg. 1985; 40: 574-81.

4. Jones EL, Weintraub WS, Craver JM, Guyton RA, Cohen CL. Coronary bypass surgery: is the operation different today? J Thorac Cardiovasc Surg. 1991; 101: 108-15.

5. Frye RL, Kronmal R, Schaff HV, Myers WO, Gersh BJ. Stroke in coronary artery bypass graft surgery: an analysis of the CASS experience: the participants in the coronary artery surgery study. Int J Cardiol. 1992: 36: 213-21.

6. Gonzales-Scarano F, Hurtig HI. Neurologic complication of coronary artery bypass grafting: case-control study. Neurology. 1981; 31: 1032-5.

7. Newman MF, Wolman R, Kanchuger M, Marschal K, Mora-Mangano C, Roach $\mathrm{G}$, et al. Multicenter preoperative stroke risk index for patients undergoing coronary artery bypass graft surgery: multicenter study of perioperative ischemia research group. Circulation. 1996; 949 (Suppl II): II-74-II-80.

8. Wolman R, Nussmeier NA, Aggarwal A, Kanchuger MS, Roach GW, Newman $M F$, et al. Cerebral injury after cardiac surgery: identification of a group at extraordinary risk. Stroke. 1999; 30: 514-22.

9. Hogue CW, Murphy SF, Schechtman KB, Davila-Roman VG. Risk factors for early or delayed stroke after cardiac surgery. Circulation. 1999; 100: 642-7.

10. Likosky DS, Marrin CAS, Caplan LR, Baribeu YR, Morton JR, Weintraub RM et al. Determination of etiologic mechanisms of stroke secondary to coronary artery bypass graft surgery. Stroke. 2003; 34: 2830-4.

11. Glance LG, Osler TM, Mukamel DB, Dick AW. Effect of complication on mortality after coronary artery bypass grafting surgery: evidence from New York State. J Thorac Cardiovasc Surg. 2007; 134: 53-8.

12. Eagle KA, Guyton RA, Davidoff R, Edwards FH, Ewy GA, Gardner TJ, et al. ACC/AHA 2004 guideline update for coronary artery bypass graft surgery: a report of the ACC/AHA Task Force on Practice Guidelines (Committee to Update the 1999 Guidelines for Coronary Artery Bypass Graft Surgery). Circulation. 2004; 110 (14): e340-437.

13. Stamou SC, Hill PC, Dangas G, Pfister AJ, Boyce SW, Dullum MKC, et al Stroke after coronary artery bypass: incidence, predictors, and clinical outcome. Stroke. 2001; 32: 1508-13.

14. Davila-Roman VG, Murphy SF, Nickerson NJ, Kouchoukos NT, Schechtman $\mathrm{KB}$, Barzilai B. Atherosclerosis of the ascending aorta is a independent predictor of long term neurologic events and mortality. J Am Coll Cardiol. 1999; 33: 1308-16.

15. Herlitz J, Wognsen GB, Haglid M, Hartford M, Emanuelsson H, Karlson BW, et al. Mortality and morbidity during a period of 2 years after coronary artery bypass surgery in patients with and without a history of hypertension. J Hypertens. 1996; 14: 309-14.

16. Assmann G, Schulte H. The prospective cardiovascular munster (PROCAM) study: prevalence of hyperlidemia in persona with hypertension and/or diabete mellitus and relationship to coronary heart disease. Am Heart J. 1988 116: 1713-24.

17. Thourani VH, Weintraub WS, Stein B, Gebhart SSP, Craver JM, Jones EL, et al Influence of diabete mellitus on early and late outcome after coronary artery bypass grafting. Ann Thorac Surg. 1999; 67: 1045-52.

18. Agmon Y, Khandheria BK, Meissner I, Schawartz GL, Petterson TM, O'Fallon M et al. Independent association of high blood pressure and aortic atherosclerosis: a population-based study. Circulation. 2000; 102: 2087-93. 